\title{
Sistema de telemetría aplicada al monitoreo de la cantidad de solución madre en sistemas de abastecimiento rural de agua
}

\section{Telemetry system applied to the monitoring of the amount of chlorine stock solution in rural water supply systems.}

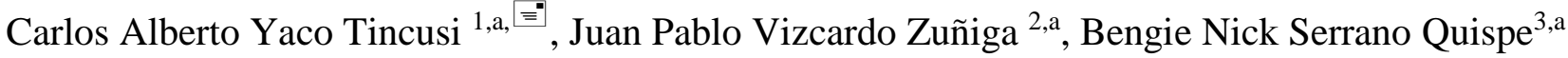 \\ 1 Universidad Andina del Cusco, Escuela Profesional de Ingeniería Civil, Cusco, Perú. \\ 2 Universidad Nacional San Antonio Abad del Cusco, Escuela Profesional de Ingeniería Electronica, Cusco, Perú \\ 3 Universidad Nacional San Antonio Abad del Cusco, Escuela Profesional de Ingeniería Informatica y de sistemas, Cusco, Perú \\ a Ingeniero \\ ”cyaco33@gmail.com
}

\begin{abstract}
Resumen
El acceso a agua clorada en zonas rurales, es una de las principales problemáticas que afronta el estado Peruano, actualmente se carecen de herramientas autónomas y remotas que permitan supervisar la cloración en los reservorios de abastecimiento de agua en las zonas rurales, en tal sentido, el objetivo del presente trabajo fue la medición continua y remota de la cantidad de solución madre en el reservorio de abastecimiento rural de agua, para tal fin, se implementó e instaló un sistema de telemetría aplicado al monitoreo de la cantidad de solución madre en el sistema de abastecimiento rural de agua del centro poblado de Chingo Grande del distrito de Saylla - Cusco. El sistema está conformado por dos módulos, el primer basado en componentes electrónicos, que es responsable de la adquisición y el envío de la cantidad de solución madre, vía red celular. El segundo de tipo informático, es el encargado de sistematizar los datos recibidos por el primer módulo, permitiendo a las instituciones responsables de la calidad del agua verificar de manera remota y continua la oportuna recarga del tanque de solución madre, así mismo, es capaz de enviar mensajes de texto SMS para alertar al responsable de la Junta Administradora de Servicios de Saneamiento. Se evaluó la operatividad del sistema, periodo durante el cual se supervisó remotamente la oportuna recarga de la sustancia madre, se constató los ciclos de recarga y se alertó oportunamente al responsable de la administración de los servicios de agua.

Palabras clave: telemetría, agua clorada, solución madre, sistemas de abastecimiento rurales
\end{abstract}

\begin{abstract}
The access to chlorinated water in rural areas, is one of the main problems faced by the Peruvian state, currently there aren't autonomous and remote tools that allow monitoring chlorination in water supply reservoirs in rural areas, in that sense, The objective of this work was the continuous and remote measurement of the amount of chlorine stock solution in the rural water supply reservoir, for this purpose, A telemetry system was implemented and installed to monitor the amount of stock solution in the rural water supply system of the populated center of Chingo Grande in the district of Saylla - Cusco. The system is made up of two modules, the first based on electronic components, is responsible for the acquisition and sending of the amount of stock solution via cellular network. The second informatic-type is responsible for systematizing the data sent by the first module, allowing the institutions responsible for water quality to remotely and continuously verify the timely recharge of the stock solution tank, also it is capable of sending SMS text messages to alert for the person in charge of the Sanitation Services Administrative Board. The operability of the system was evaluated, during which the timely recharge of the parent substance was monitored remotely, the recharge cycles were verified and the person responsible for the administration of the water services was duly alerted.

Keywords: Telemetry, chlorinated water, chlorine stock solution, rural water supply systems.
\end{abstract}

Citar como: Tincusi, CAY., Zuñiga, JPV., Quispe, BNS. (2019). Sistema de telemetría aplicada al monitoreo de la cantidad de solución madre en sistemas de abastecimiento rural de agua. Rev Yachay, 8(1),553-558.

Recibido: 30-07-2019; Aceptado 12-10-2018: Publicado: 12-12-2019

\section{Introducción}

El acceso al agua clorada es algo que damos por sentado en grandes ciudades y capitales de provincia, sin embargo, en el ámbito rural el escenario es completamente diferente. Si bien a nivel nacional el 74.4\% de la población rural tiene acceso al agua, menos del 3\% consume agua clorada (INEI, 2019). La situación en la región Cusco es similar, contando con una cobertura de 93.3\%, pero sólo el 11\% accede al 
agua clorada. Y esto a pesar de que en la región tenemos alrededor de 5000 sistemas de abastecimiento de agua gestionados mediante las Juntas Administradoras de Servicios de Saneamiento (DATASS, 2018). El método predominante para la cloración de sistemas de abastecimiento de agua a nivel rural es el denominado sistema de goteo, el cual consiste en el vertido de cloro en altas concentraciones almacenado en un tanque de plástico (solución madre) hacia el reservorio principal.

Uno de los principales problemas en el consumo de agua clorada es la inoportuna dotación y recarga de cloro en los tanques de solución madre por parte del responsable, el cual se puede resolver si somos capaces de medir de forma continua y remota la cantidad de solución madre. Por lo que nos planteamos la siguiente interrogante: ¿Cómo medir de forma continua y remota la cantidad de solución madre en reservorios rurales?

En tal sentido, el objetivo de la presente investigación fue la medición continua y remota de la cantidad de solución madre en reservorios rurales y alertar oportunamente a los encargados de las JASS para la recarga correspondiente. Adicionalmente, debemos considerar que a nivel nacional no se cuenta con medios de verificación remotos que permitan realizar dicha labor

\section{Materiales y métodos}

La presente investigación comprende la implementación de un sistema de medición remota de la cantidad de solución madre en reservorios rurales, en adelante denominado "Sistema de telemetría aplicada al monitoreo de la cantidad de solución madre en reservorios rurales". Para el diseño se consideró el entorno y las condiciones de los sistemas de abastecimiento de agua en zonas rurales de la región Cusco como el tipo el tipo de sistema de cloración empleado y la inexistencia de energía eléctrica.

El tipo de investigación fue aplicada y el nivel de investigación, experimental; debido a que con esta investigación se logró construir un sistema de telemetría que permite monitorizar remotamente el nivel de sustancia madre en el tanque de cloración.

En cuanto a la metodología, la investigación se realizó en tres fases: la primera es la fase teórica en la cual se recopiló información referente al tema en bases de datos como: Scopus, Web of Science y Google Academic. En la segunda fase se procedió a realizar entrevistas a los agentes involucrados y relacionados con la cloración del agua en ámbitos rurales: integrantes de las JASS, a los responsables de las Áreas Técnicas Municipales (ATM), al encargado del Fondo al Estimulo del Desempeño (FED) de la Dirección Regional de Vivienda Construcción y Saneamiento (DRVCS) del Gobierno Regional del Cusco y, finalmente, al responsable del Centro de Atención al Ciudadano del Ministerio de Vivienda Construcción y Saneamiento, con el objetivo de recabar información respecto al funcionamiento de las JASS, ATM y los procesos necesarios para dotar de cloro al sistema de cloración. En la Tercera fase se realizó el diseño del sistema de telemetría, el cual está conformado por:

a) Subsistema de monitoreo de cantidad de sustancia madre

b) Subsistema informático

Subsistema de monitoreo de cantidad de sustancia madre, es el encargado de medir la cantidad de sustancia madre presente en el tanque de cloración y enviar la información a la plataforma informática, utilizando para ello la red celular, para este fin el subsistema de monitoreo está conformado por las siguientes etapas:

i. Etapa de suministro de energía, conformado por un panel solar, controlador y batería; esta etapa se encarga de suministrar energía al subsistema.

ii. Etapa de Comunicación, conformado por un módulo electrónico; etapa encargada de la transmisión de los datos a la plataforma informática utilizando la red celular.

iii. Etapa de adquisición de nivel del tanque, conformado por un sensor ultrasónico; etapa encargada de medir la altura del tanque de solución madre.

iv. Etapa de Procesamiento de datos, conformado por un módulo electrónico, esta etapa se encarga de procesar las señales del sensor ultrasónico y enviar los datos a través de la etapa de comunicación. 


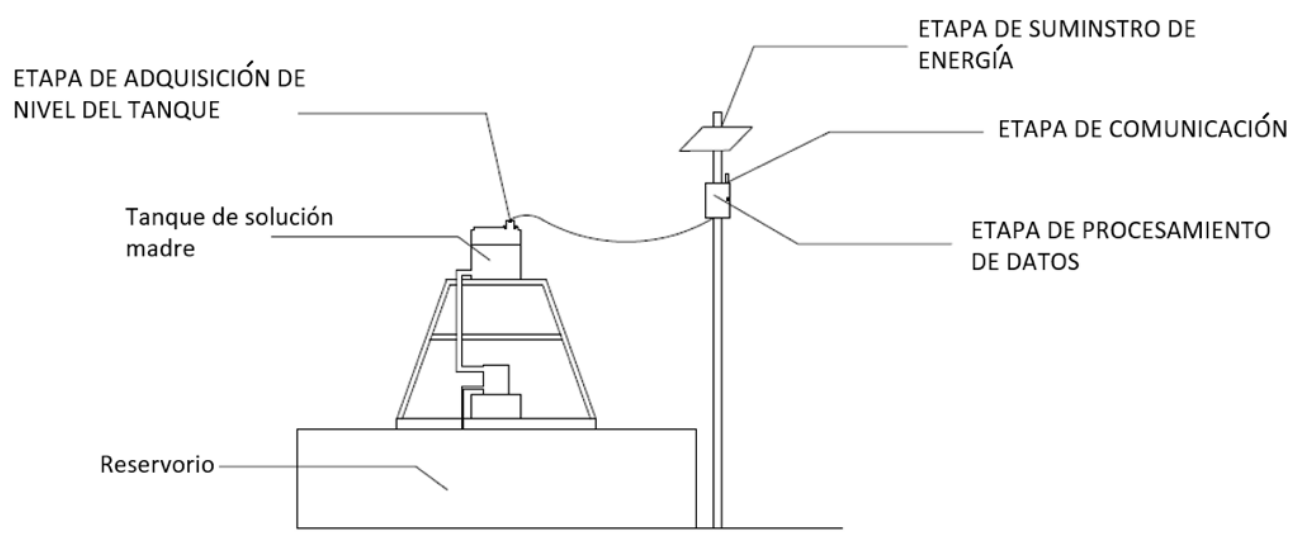

Figura 2. Subsistema de monitoreo de cantidad de sustancia madre

Subsistema informático, encargado de sistematizar la información de la cantidad de solución madre proveniente del subsistema de monitoreo, permitiendo acceder a datos remota e instantáneamente y así, determinar la oportuna recarga del tanque de solución madre, también permite detectar la obstrucción del conducto que lleva la solución madre hacia el reservorio principal. El subsistema informático es encargado también de enviar los mensajes de texto SMS a los responsables de la JASS, ATM u otros según configuración. Este subsistema está instalado en servidores en la nube, por lo que puede accederse desde cualquier dispositivo con conexión a internet

\section{Resultados}

\section{Subsistema de monitoreo de cantidad de sustancia madre}

Este subsistema se instaló en el reservorio rural ubicado en el centro poblado de Chingo Grande del distrito de Saylla.

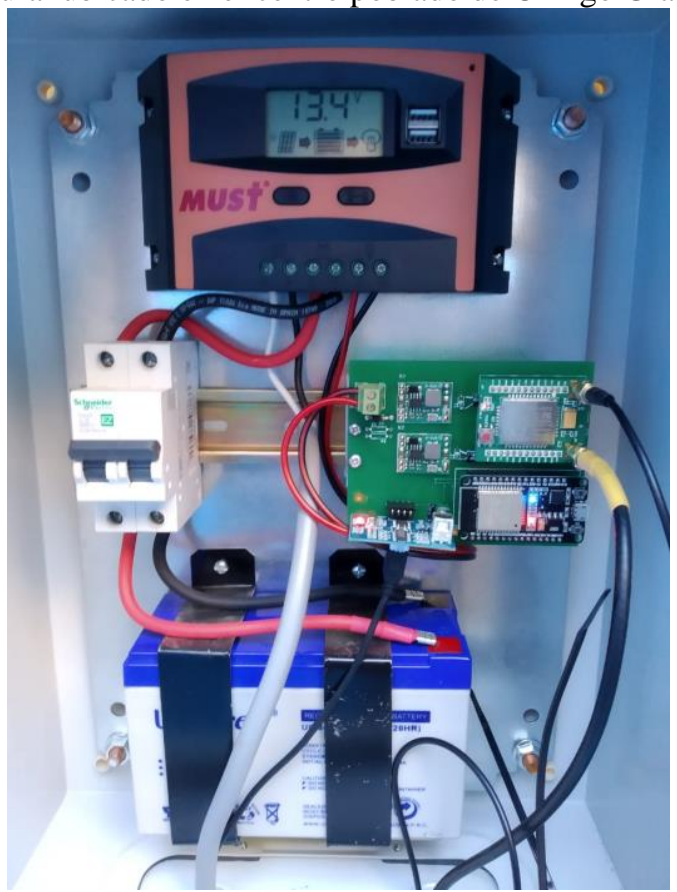

Figura 3. Interior de parte del subsistema de monitoreo de cantidad de sustancia madre 


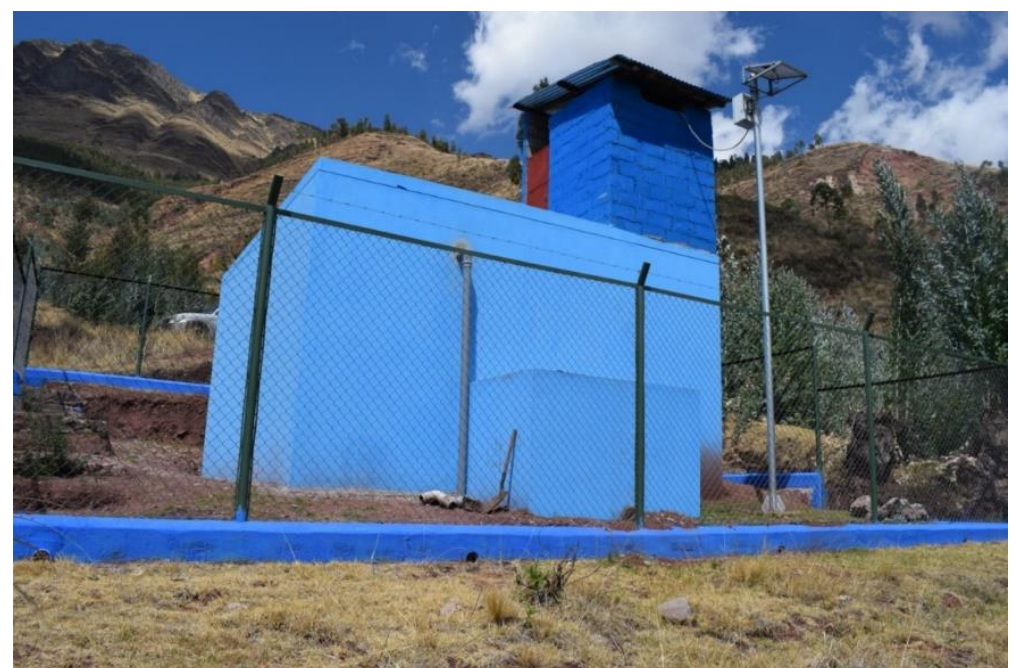

Figura 4. Vista exterior del subsistema de monitoreo de cantidad de sustancia madre instalado en Saylla.

\section{Plataforma informática}

Es accesible mediante cualquier dispositivo con conexión a internet, a través de la página web: https://aguaregioncusco.com/

\section{Permite:}

a) Visualizar en un mapa los reservorios que cuenten con el subsistema de monitoreo continuo y ver el reporte de la cantidad de solución madre del tanque de cloración.

b) Configurar el envío mensaje de texto a los responsables de la JASS, ATM u otros responsables alertando sobre la cantidad de solución madre presente en el sistema de cloración.

c) Historial gráfico de la carga y descarga de la solución madre en los sistemas de cloración.

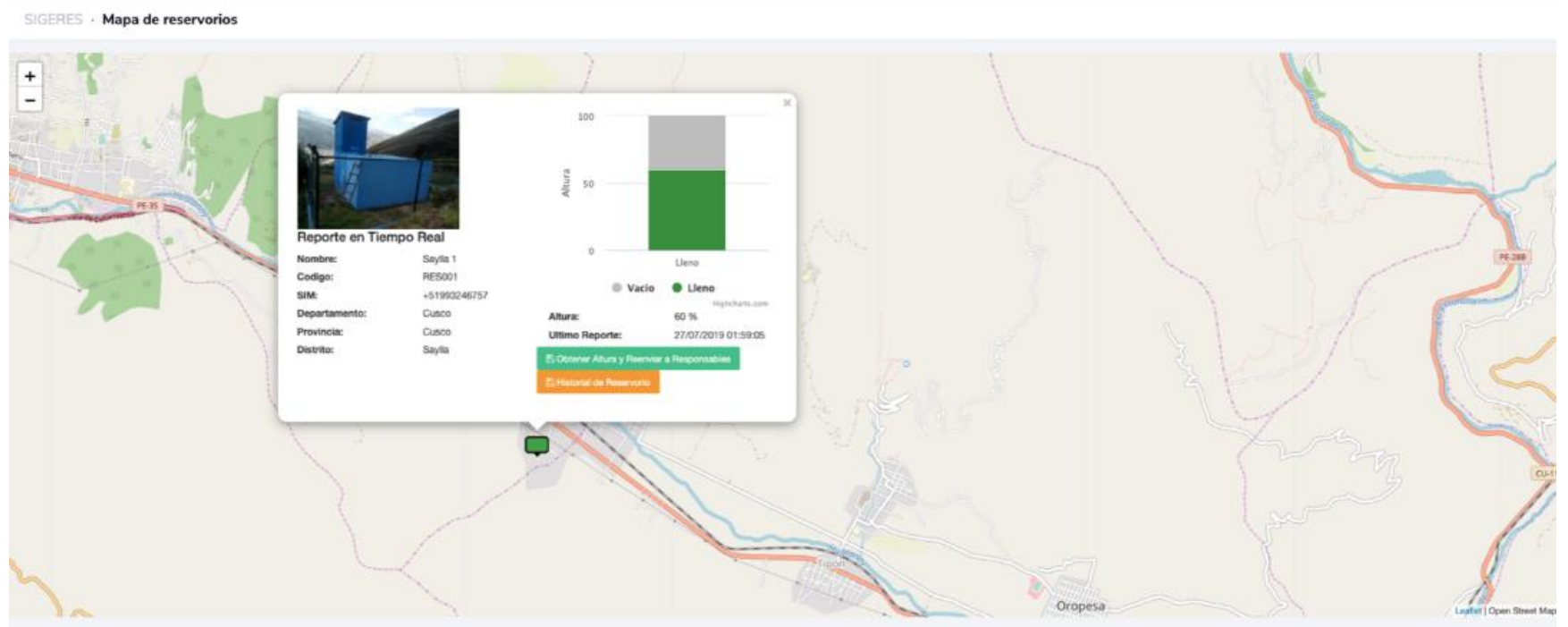

Figura 5. Captura de pantalla de la plataforma informática. 


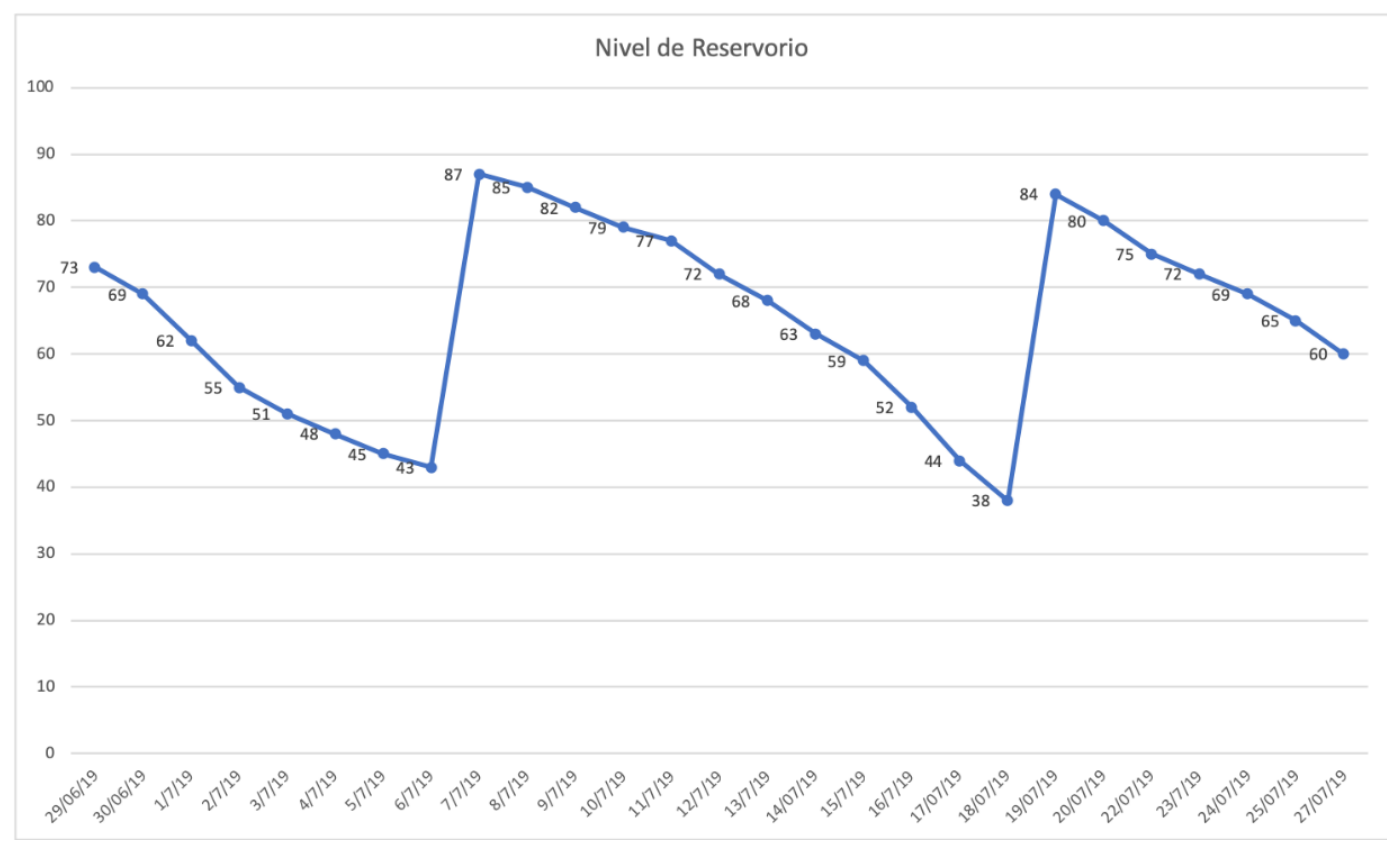

\section{Discusión}

Figura 6. Historial de datos de carga y descarga de sustancia madre en el sistema de cloración

En la presente investigación se logró monitorizar la cantidad de solución madre en el sistema de cloración rural, siendo este un primer paso para garantizar el acceso a agua clorada. A nuestro criterio, queda pendiente determinar y monitorear la cantidad de cloro presente en el agua, que según la normativa vigente debe de estar entre 0.5 a $1 \mathrm{ppm}$ (partes por millón).

Durante el periodo de prueba, el sistema alerto por mensajes de texto al encargado de la JASS para la recarga oportuna de la sustancia madre, garantizándose que, durante todo este periodo, la población fuera dotada de agua clorada, tal como se puede verificar en los datos, es decir no existió un lapso de tiempo, en el cual el sistema de abastecimiento no cuente con sustancia madre, lo cual concuerda con las mediciones obtenidas por la Dirección Regional de Salud (DIRESA).

\section{Conclusiones}

Primera. Al poder monitorizar la cantidad de solución madre en los sistemas de cloración, se puede alertar oportunamente a los responsables de las JASS una vez que la solución madre esté cerca de agotarse, permitiendo una recarga oportuna.

Segunda. Se logró diseñar y construir un sistema de telemetría completamente funcional capaz de monitorizar la cantidad de solución madre en un sistema de abastecimiento de agua rural típico de la región Cusco

\section{Referencias bibliograficas}

Bertram, J. (2012). Clientes de servicios web seguros en dispositivos móviles. Procedia Computer Science.

Bolaños, J. (2013). Implicaciones del monitoreo periódico de la calidad del agua potable en el cantón de Grecia, Alajuela, Costa Rica. Revista Electrónica de las Sedes Regionales de la Universidad de Costa Rica Recuperado el 03 de mayo de : https://www.redalyc.org/pdf/666/666294 46003.pdf
DATASS (2018) Diagnóstico sobre el abastecimiento de agua y saneamiento en el ámbito rural. Recuperado el 15 de agosto de: https://datass.vivienda.gob.pe/

García Muñoz, C. (2016). Informática IES Gonzalo Nazareto. Recuperado de Sharding in MongoDB: http://informatica.gonzalonazareno.org/p royectos/2016-
17/Cluster_Sharding_MongoDB_Carlos _Garcia.pdf

Gracia, O. (2014). Aplicación de tiempo real con node.js . Northware.

Instituto Nacional de Estadística e Informática. (2017). Encuesta Nacional de Programas Presupuestales. Recuperado 16 de Abril de 2019 de: https://webinei.inei.gob.pe/anda_inei/ind ex.php/catalog/614 
INEI - Instituto Nacional de Estadistica e Informatica(2019), Perú formas de acceso al agua y saneamiento basico Recuperado el 15 de Agosto de : https://www.inei.gob.pe/media/MenuRe cursivo/boletines/boletin-el-agua-3.pdf

Kozlowski, P., \& Darwin, B. (2013). Mastering Web Application Development with AngularJS. Packt Publishing, Limited.

Ministerio de Salud - Perú. (2019) Desinfección de sistemas, caracterización de fuentes de agua y cloración de agua para el consumo humano. Recuperado 30 de junio de 2019 de: https://www.mef.gob.pe/contenidos/pres u publ/migl/metas/taller PI meta35 2.p $\underline{\mathrm{df}}$

Ministerio de Vivienda, Construcción y Saneamiento (2019), DATASS. Recuperado 15 de Abril de 2019 de: https://datass.vivienda.gob.pe/

Miranda M., Aramburú A., Junco J. y Campos M. (2010). Situación de la calidad de agua para consumo en hogares de niños menores de cinco años en Perú, 2007-
2010. Revista Peruana de Medicina Experimental y Salud Pública. Recuperado el 30 de abril de: https://www.scielosp.org/scielo.php?pid $=\mathrm{S} 1726$ -

$46342010000400003 \&$ script=sci_arttext \&thng $=\mathrm{pt}$

MQTT ORG. (2017). Frequently Asked Questions. Recuperado el 25 de abril del 2018 de Mqtt.org: http://mqtt.org/faq 